\title{
XVII. The Conductivity produced in gases by the motion of negatively charged ions
}

\author{
John S. Townsend M.A.
}

To cite this article: John S. Townsend M.A. (1901) XVII. The Conductivity produced in gases by the motion of negatively charged ions, Philosophical Magazine Series 6, 1:2, 198-227, DOI: 10.1080/14786440109462605

To link to this article: http://dx.doi.org/10.1080/14786440109462605

曲 Published online: 16 Apr 2009.

Submit your article to this journal \lceil

山 Article views: 23

Q View related articles $\square$

4 Citing articles: 33 View citing articles 5 
values of $r$, the square of $\mathrm{A}$ is seen to be negiigible in comparison with unity.

Now $\mathrm{B}$ is less than $\mathrm{A}$, for $k$ is less than 1 , and $a+x$ is greater than $a$. Hence, $\mathrm{B}^{2}$ and $\mathrm{AB}$ are negligible in comparison with 1 , and our equation for maximum and minimum reduces to the form

$$
\cos \frac{2 \pi x}{\lambda}= \pm 1
$$

whence

$$
x=\frac{n \lambda}{2}
$$

where $n$ is any integer.

Whence it follows that with the arrangement of apparatus adopted in the experiment on stationary wave and index of refraction, the diminished amplitude of the reflected wave and the absorption by the dielectric placed between the oscillator and the reflector could have no appreciable effect on the position of the maxima and minima.

Jefferson Physical Laboratory,

Harvard University, Cambridge, Mass.

XVII. The Conductivity produced in Gases by the Motion of Negatively charged Ions. By JoHN S. Townsend, M.A., Wykeham Professor of Physics, Oxford*.

1. TN a paper published in 'Nature,' vol. lxii. August 9th, 1900 , I gave a brief description of some experiments which showed that negatively charged ions, moving through a gas, produce other ions, although the force acting on them is very small compared with the force necessary to produce the ordinary vacuum-tube or spark-discharges. The present paper contains a more complete account of the principal experiments and also some investigations founded on the theory to which I have been led by the experimental results.

In all the experiments with which we are here concerned, a number of ions are generated in the gas by some external source, such as Röntgen or Becquerel rays. The nature of the conductivity produced by these rays has been investigated by several physicists, and it has been generally concluded that when the electric force is increased, the conductivity approaches a maximum. Thus, in the paper $t$ published on this subject by Professors J. J. Thomson and E. Ruthertord, it is stated that "for a given intensity of radiation, the

* Communicated by the Author.

† Prot. J. J. Thomson and Mr. Rutherford, Phil. Mag. Nov. 1896. 
current through the gas does not exceed a certain maximum value whatever the electromotive force may be."

Numerous experiments have also been made with electrode, of various shapes, and it was found that the conductivity is not altered when the electromotive force is reversed.

From the experiments which are described in the present paper, it will be seen that the relation between current and (l)ectromotive force is not characterized by either of these properties when the conductivity is produced in a gas at a low pressure. There is no critical pressure or force at which discontinuity occurs; but, as the pressure is reduced, the comnexion between current and electromotive force gradually loses the simple properties which hold at high pressures.

At low pressures the current may be considered to pass through three stages as the electromotive force is increased. In the first stage, the current increases with the electromotive force; in the second stage, the current remains practically constant and shows only small variations for comparatively large changes in the force; in the third stage, the current rapidly increases with the electromotive force. Also, for certain shapes of electrodes, a great difference can be produced in the conductivity by reversing the electromotive force. This is particularly noticeable when the current passes between two electrodes, one of which surrounds the other; but when parallel plates are used, the conductivity shows only slight alterations when the direction of the current is changed. In the latter case there would be no alteration whatsoever if the two plates were made of the vame metal. We shall therefore begin by describing the nature of the conductivity between two parallel plates when the gas between them is at a low pressure.

2. The apparatus used for the investigation is shown in fig. 1 (p. 200), and consists of two parallel plates inside an air-tight vessel connected to a Töpler pump. The lower plate A was a thick sheet of zinc with a circular window $W$ which was covered with a disc of thin aluminium. The junction between the aluminium and the zinc was sealed with elastic glue, so as to make the joint air-tight.

A sheet of aluminium foil was stretched over the surface of the zinc plate so that a plane face should be presented to the movable brass plate $\mathrm{B}$. The tube $\mathrm{T}$, by which the vessel was exhausted, was soldered to the back of the brass plate.

The brass plate $B$ was 10 centimetres in diameter, and the circular aperture $\mathrm{W}$ was 4 centimetres in diameter.

The neck of the glass covering $\mathrm{C}$ was closed with an 


\section{2(1) Prof. Townsend on the Conductivity produced in}

ebonite plug through which a brass tube $U$ was passed, and the junction between the tube and the ebonite was made airtight. The tube $T$ fitted exactly into the tube $U$ and could be moved up and down in it; by this means the distance

Fig. 1.

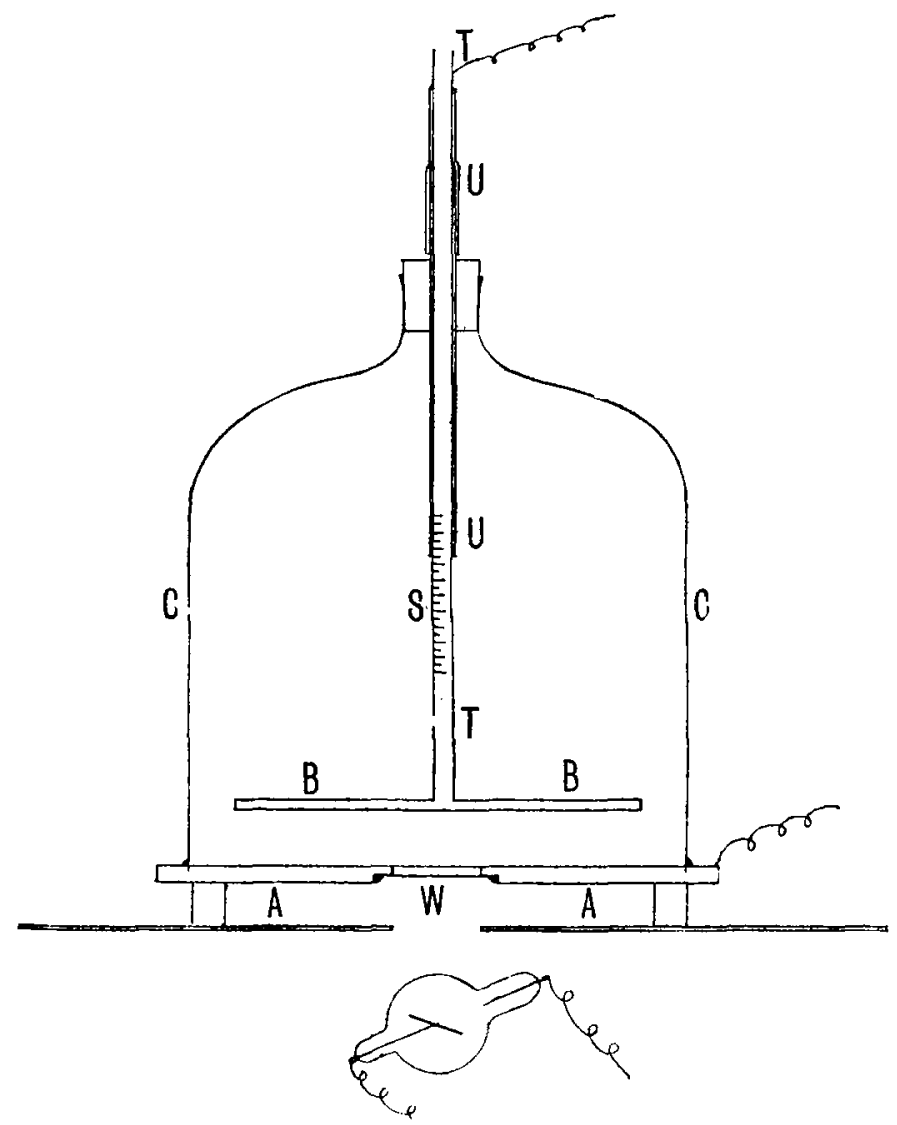

between the two plates $A$ and $B$ could be varied, and a scale $S$ ruled on the tube $T$ gave a means of determining the distance between the plates. A piece of rubber tubing was put over the upper end of the tube $\mathrm{U}$, so as to prevent air getting into the apparatus between the two tubes.

The pressure of the gas inside the apparatus was found by a MacLeod gauge.

The electrical connexions were made in the usual manner. The rine plate A, which was supported on ebonite legs, was 
connected to one terminal of a battery of lead cells, the other terminal of which was connected to earth. The upper plate $\mathrm{B}$ was connected by the tube ' $\mathrm{I}$ to one pair of quadrants of an electrometer, the other pair of quadrants and the case being connected to earth. The wire joining the tube $\mathrm{T}$ to the quadrants passed along the centro of brass tubing which was connected to earth, and thus formed a screen for the wire.

Strips of tinfoil were gummed round the glass vessel. C both on the inside and the outside, and were kept at zero potential by means of earth connexions. The lower edges of the bands were within 2 centimetres of the zine plate, so that no charge could leak from the plate $A$ over the surface of the glass and influence the potential of the upper plate and the tube T.

The ebonite legs supporting the apparatus rested on a box, covered with lead, containing a Röntgen-ray focus-bulb, and a Ruhmkorff coil. A circular hole was cut in the lead covering, and the bulb was placed so that the platinum anode should be vertically under the centre of the aperture.

The direct rays traversed the space between the two plates, above the aluminium window; and the insulated dise B, which was of thick brass, prevented the radiation from penetrating into the upper part of the apparatus. At the sime time the brass plate gave out a secondary radiation which increased considerably the ionization between the plates.

3. The experiments were conducted in the following manner. The wire connecting the upper plate to the quadrants was put to earth and the lower plate was raised to the required potential; the upper plate was then insulated. The deflexion on the electrometer-scale, produced when the rays were acting for 10 seconds, was observed. The electrometer showed no leak until the bulb was excited; and after the rays were stopped, the electrometer reading remained perfectly steady, showing that the current between the plates does not continue after the rays have ceased to act.

The connexion between current and electric force was thus obtained for five different pressures, and the numbers obtained are represented graphically by the accompanying curves, figs. $2,3,4,5$, and 6. The current between the plates varies slightly, in magnitude, on reversing the electromotive force. The mean of the two currents obtained by reversing the electromctive force is represented by the curves. The difference in conductivity is generally shall and could have been aroided by using two aluminium plates; but the advantage thus gained would not compensate for the loss Phil. Mag. S. 6. Vol. 1. No. 2. Feb. 1901. 
202 Prof. Townsend on the Conductivity produced in

of conductivity arising from the large secondary effect due to the brass. In Section 9, an account is given of the effect produced by reversing the electromotive force between the plates.

Fig. 2.-Pressure 4.13 $\mathrm{mm}$.

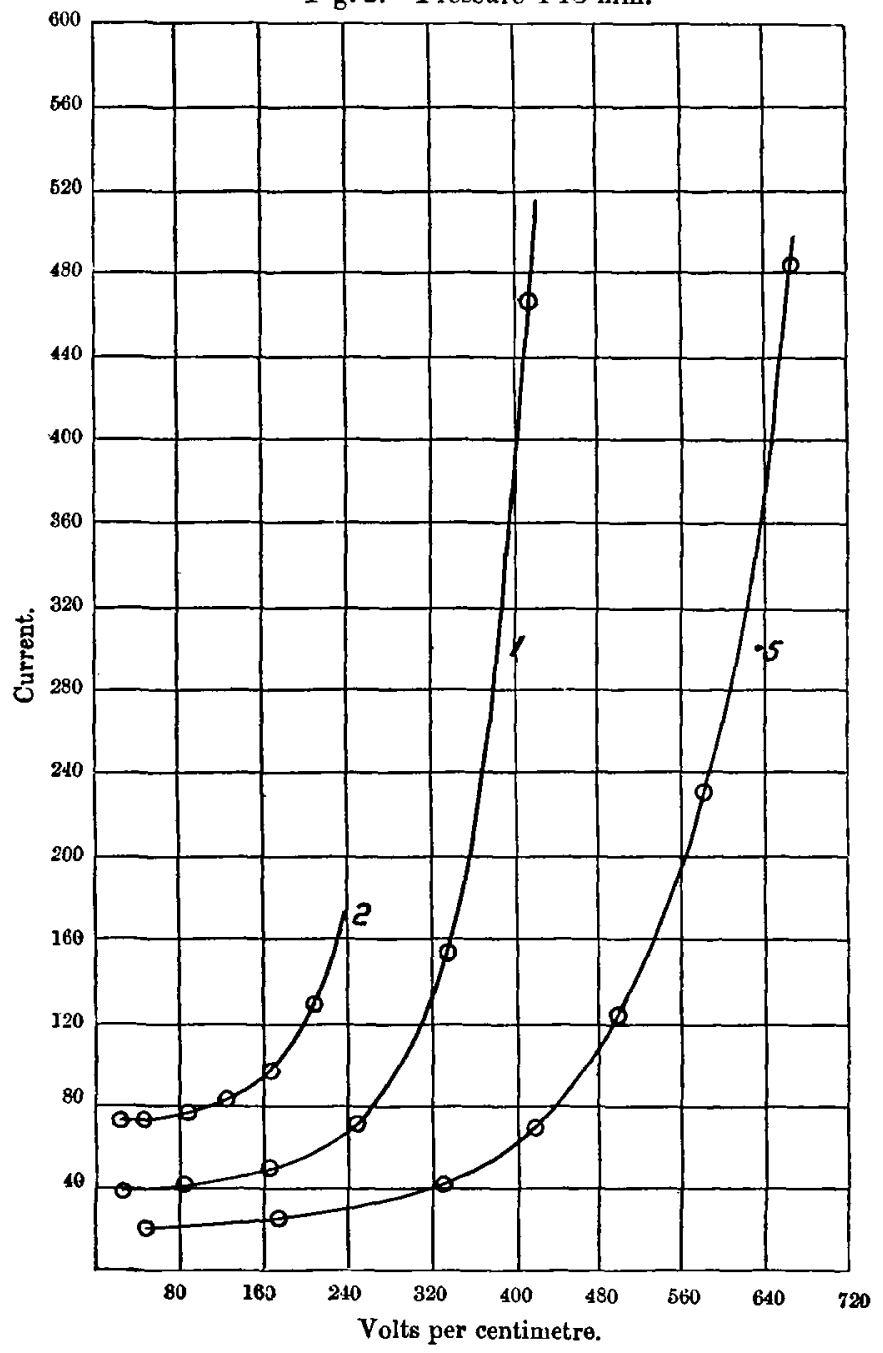

Three seis of observations were made at each pressure for distances of 5,1 , and 2 centimetres between the plates. The curves corresponding to these distanees are marked $\cdot 5,1$, 
Gases by the Motion of Negatively charged Ions. 203 and 2, respectively. The forces $\mathrm{X}$ are given in volts per centimetre [(potential of $\mathrm{A}) \div$ (distance between the plates)]. The unit of current is $1 \cdot 410^{-13}$ amperes. The curves have

Fig. 3.-Pressure 2·i2 millim.

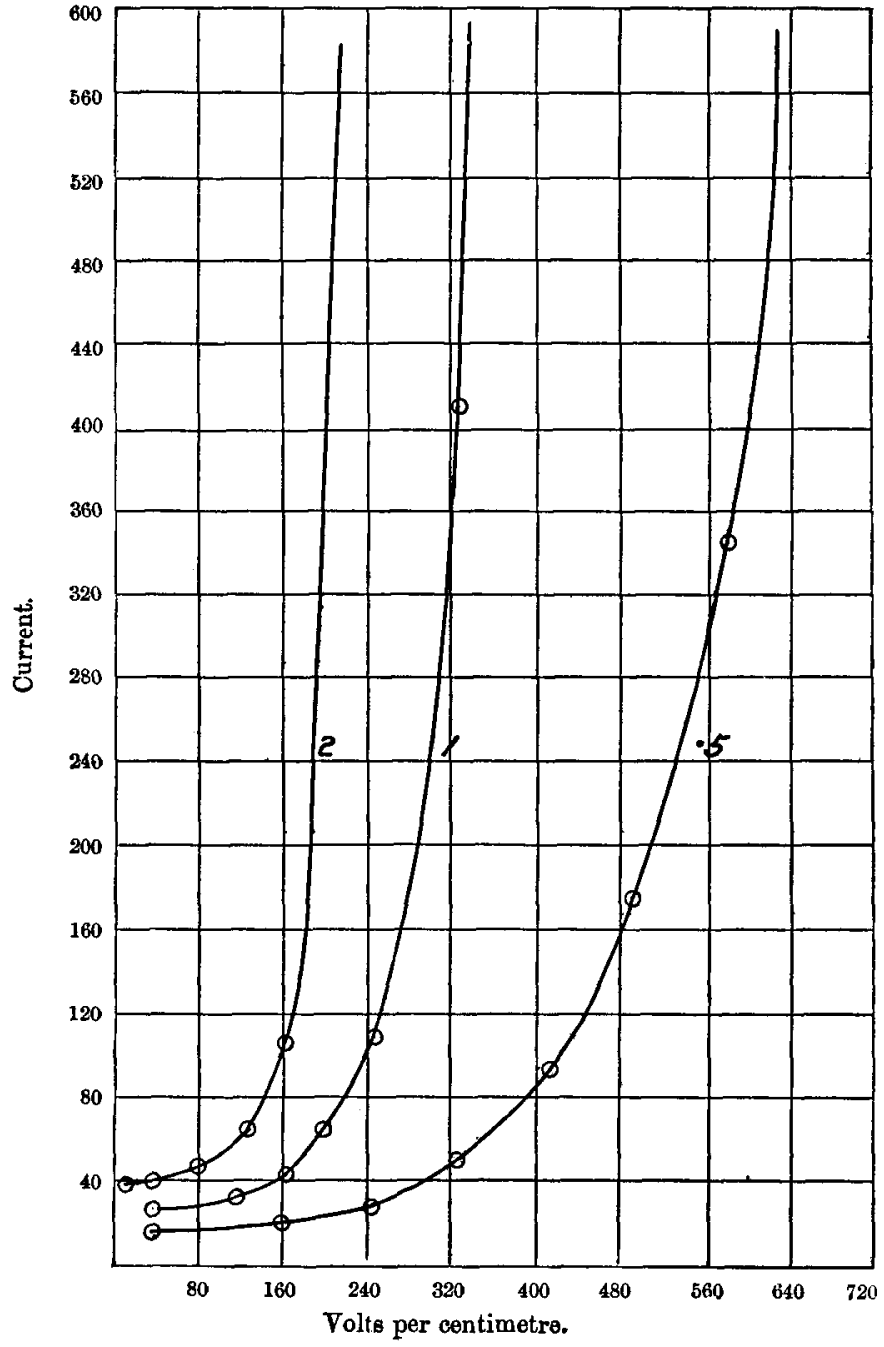

not been traced back to the origin, as we are at present only concerned with the connexion between current and electric force when the potential.difference between the electrodes P 2 
204 Prof. Townsend on the Conductivity produced in

exceeds 10 or 20 volts. The curves show that the current increases, sometimes very rapidly, after the second stage (to which I have referred) is passed.

Fig. 4.-Pressure $1 \cdot 10 \mathrm{~mm}$.

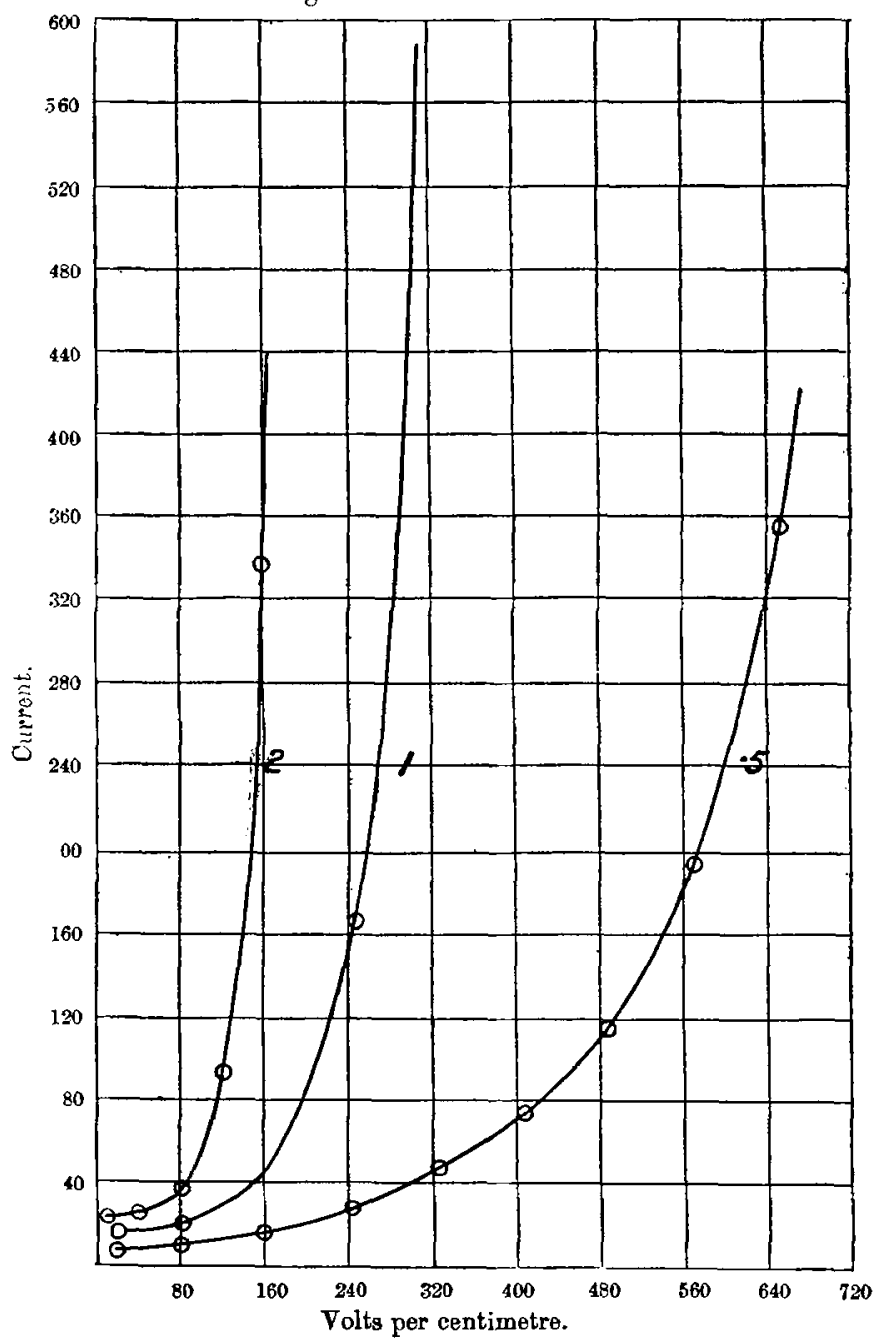

These experimental results are completely accounted for if we consider that all ions produced by the rays, including the secondary rays, are collected on the electrodes when the electromotive force is small and the current independent, 
Gases by the Motion of Negatively charged Ions. 205 practically, of the electromotive force ; and that the negative ions produce others by collisions with the molecules of the gas when the electric force is increased, the new negative ions thus produced having the same property as the negative ions produced initially by the rays.

Fig. 5.-Pressure $\cdot 332 \mathrm{~mm}$.

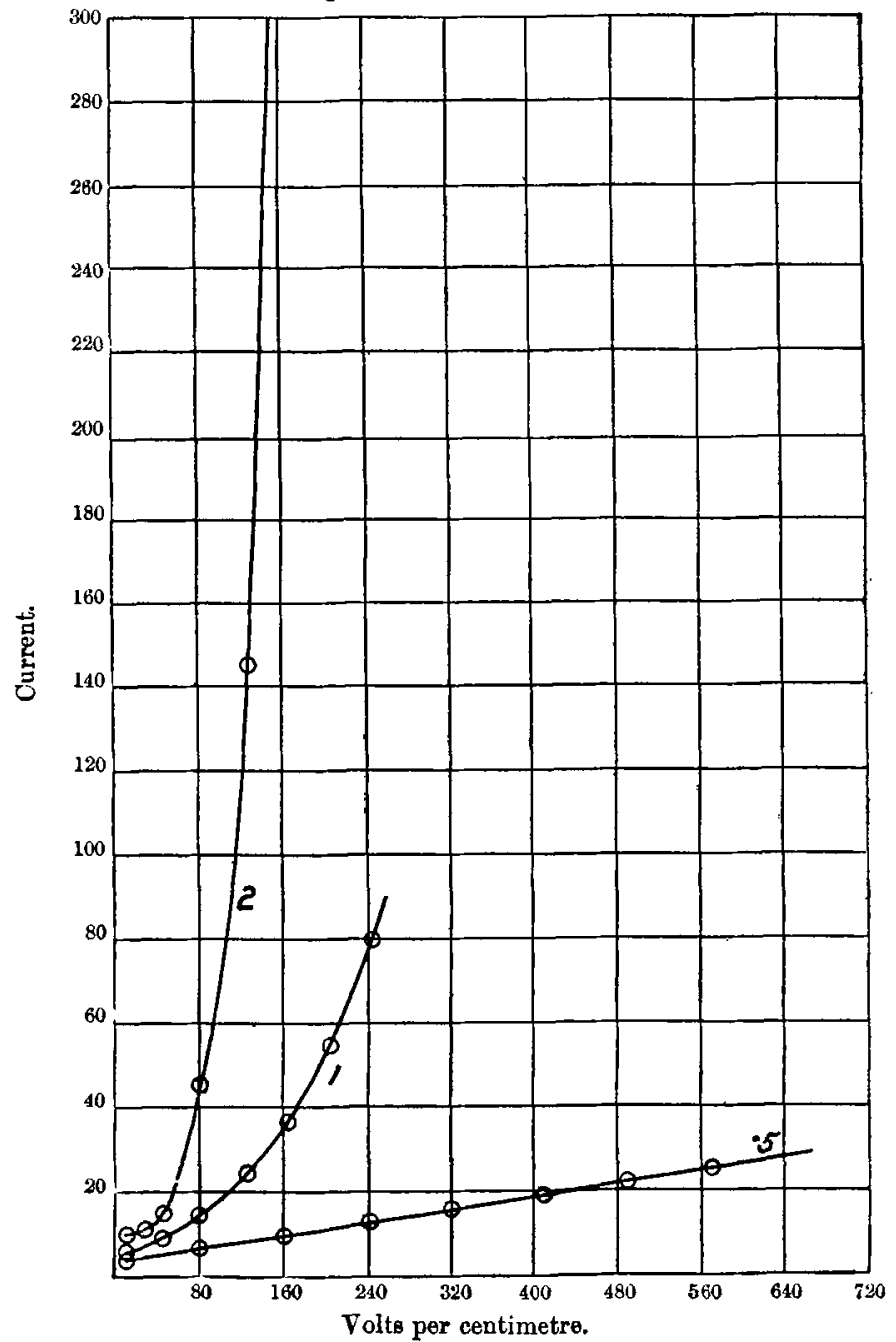

If both positive and negative ions produced others by collisions with the molecules, it is evident that the current 
would not cease when the rays are stopped. We must therefore attribute the increase in conductivity to ions of one kind; and it can be clearly shown from experiments with one electrode inside the other, that it is the negative ions which produce the large increases in conductivity.

Fig. 6.-Pressure $171 \mathrm{~mm}$.

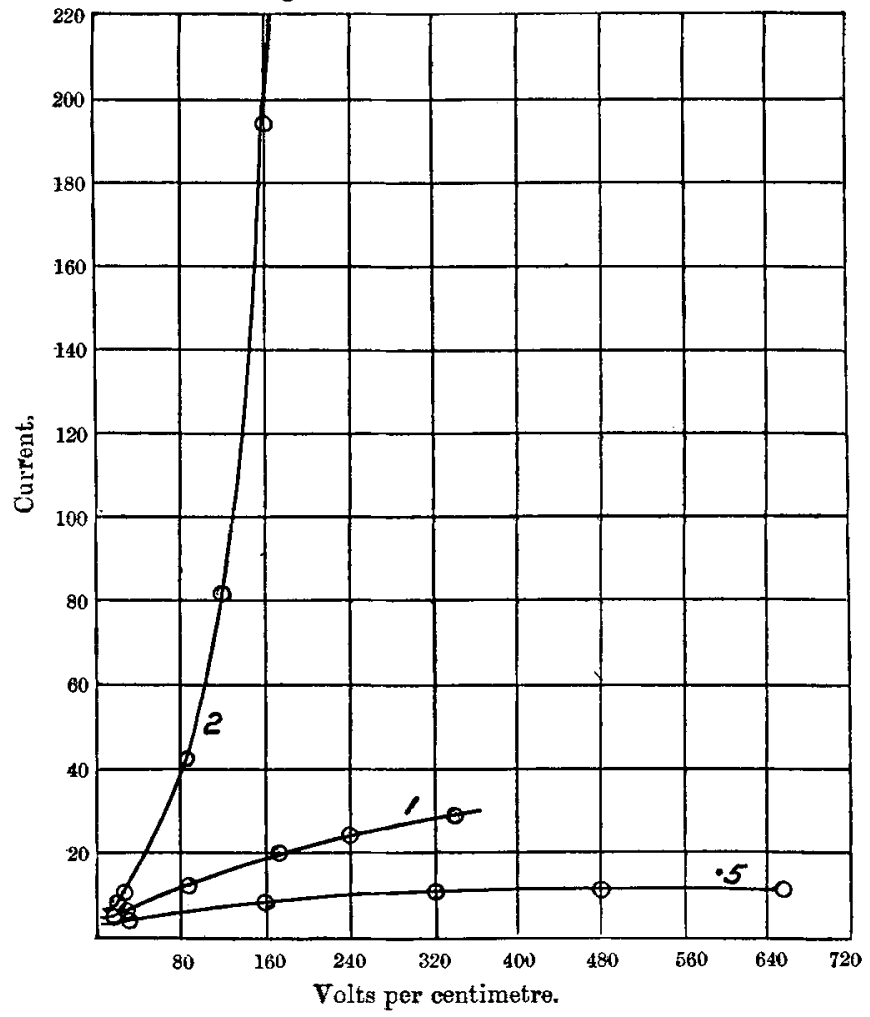

4. The first step towards an explanation of these results is to find from the curves the number, $\alpha$, of ions of one kind that a single negative ion will generate in moving under an electric force through a centimetre of the gas. The number of positive ions which are generated is equal to the number of negative ions, so that in what follows we need consider only the negative ions ; it being understood that when a negative ion is produced, a positive ion is also produced.

Let a force $X$ be applied to $N_{0}$ negative ions in a gas at pressure $p$ and temperature $t$. Let $\hat{\mathrm{N}}$ be the total number of negative ions after the $\mathrm{N}_{0}$ ions have travelled a distance $x$. 
Gases by the Motion of Negatizely charged Ions. 207

The new negative ions travel with the same velocity as the original $\mathrm{N}_{n}$ ions, so that all the negative ions will be found together during the motion. The number of negative ions produced by $N$ ions travelling through a distance $d x$ will be $\alpha \mathrm{N} d x$; where $\alpha$ is a constant depending on $\mathrm{X}, p$, and $t$.

Then

Hence

$$
d \mathrm{~N}=\alpha \mathrm{N} d x .
$$

$$
\mathrm{N}=\mathrm{N}_{0} \epsilon^{\alpha x} \text {. . . . . . . }
$$

Let $n_{0} l$ negative ions be distributed uniformly between two plates at a distance $l$ apart, and let the force $X$ be applied to them.

The number of ions $\mathrm{C}$ that arrive at the positive plate is

$$
\mathrm{C}=\int_{0}^{l} n_{0} \epsilon^{\alpha x} d x=\frac{n_{0}}{\alpha}\left(\epsilon^{\alpha l}-1\right)
$$

and the ratio of this number to the original number is

$$
\frac{\mathrm{C}}{n_{0} l}=\frac{1}{a l}\left(\epsilon^{\alpha l}-1\right) \text {. }
$$

Hence if $\mathrm{C}_{0}$ denotes the number of negative ions produced by the primary and secondary rays the total current in the gas is

$$
\mathrm{C}=\frac{\mathrm{C}_{0}}{\alpha l}\left(\epsilon^{a t}-1\right) . \quad \ldots . . .
$$

$\mathrm{C}_{0}$, the current when no ions are being produced by collisions, is represented in each curve by the $(y)$ ordinate at the point where the tangent to the curve is practically parallel to the

axis of $\mathrm{X}$. calculations it can be shown that equation (2) completely explains the relative shapes of the three curres in each diagram. The value of $\alpha$ can be found by substituting the experimentally determined ratio, $\frac{\mathrm{C}}{\mathrm{C}_{0}}$, and distance between
the plates $l$ in equation (2).

The small values of $\alpha$ are determined, most accurately, from the curves corresponding to $l=2$; but in the following tables some of the values have been obtained from two eurves, and the agreement between these numbers shows that the effect of separating the plates is completely explained by the theory. The tables give the values of $a$ for different forces $\mathrm{X}$ : the determinations made from curves corresponding to distances between the plates of 2,1 , and 5 centimetre are given in the second, third, and fourth columns respectively. A separate table is given for each pressure. 
TABLE I.

Pressure $4 \cdot 13$ millims.

\begin{tabular}{|c|c|c|c|c|}
\hline X. & $l=2$. & $l=1$. & $l=\cdot 5$. & $\begin{array}{l}\text { Calcu- - } \\
\text { lated. }\end{array}$ \\
\hline 120 & $\cdot 13$ & & ... & $\cdot 135$ \\
\hline 160 & $\cdot 28$ & $\cdot 30$ & $\ldots$ & 31 \\
\hline 200 & 50 & 51 & & \\
\hline 240 & $\ldots$ & .99 & & .95 \\
\hline $3: 0$ & $\ldots$ & $2 \cdot 1$ & $2 \cdot 2$ & 1.95 \\
\hline 400 & $\ldots$ & $3 \cdot 6$ & $3 \cdot 6$ & \\
\hline 480 & $\ldots$ & $\ldots$ & $5 \cdot 3$ & $5 \cdot 0$ \\
\hline 560 & $\cdots$ & $\ldots$ & $7 \cdot 1$ & \\
\hline 640 & $\ldots$ & $\cdots$ & 8.9 & 87 \\
\hline
\end{tabular}

TABLE III.

Pressure $1 \cdot 10$ millim.

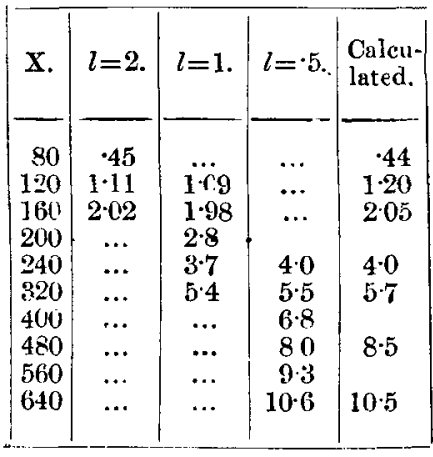

TABLE II.

Pressure 212 millims.

\begin{tabular}{|r|c|c|c|c|}
\hline $\mathbf{x}$. & $l=2$. & $l=1$. & $l=\cdot 5$. & $\begin{array}{c}\text { Calcu-u- } \\
\text { lated. }\end{array}$ \\
\hline 80 & $\cdot 13$ & $\ldots$ & $\ldots$ & $\cdot 14$ \\
120 & $\cdot 42$ & $\cdot 40$ & $\ldots$ & $\cdot 44$ \\
160 & $\cdot 90$ & $\cdot 90$ & $\cdots$ & $\cdot 91$ \\
200 & $\ldots$ & $1 \cdot 60$ & & \\
240 & $\ldots$ & $2 \cdot 35$ & $2 \cdot 35$ & $2 \cdot 4$ \\
320 & $\ldots$ & $4 \cdot 1$ & $4 \cdot 0$ & $4 \cdot 2$ \\
400 & $\ldots$ & $\ldots$ & $6 \cdot 0$ & \\
480 & $\ldots$ & $\ldots$ & $7 \cdot 8$ & $8 \cdot 0$ \\
560 & $\ldots$ & $\ldots$ & $9 \cdot 4$ & \\
640 & $\ldots$ & $\cdots$ & $10 \cdot 8$ & $11 \cdot 3$ \\
\hline
\end{tabular}

TABLE IV.

Pressure $\cdot 385$ millim.

\begin{tabular}{|c|c|c|c|c|}
\hline $\mathrm{X}$. & $l=2$. & $l=1$. & $l=5$. & $\begin{array}{l}\text { Calcu- } \\
\text { latedl. }\end{array}$ \\
\hline 40 & .34 & & & $\cdot 35$ \\
\hline 80 & $1 \cdot 3$ & $\ldots$ & & 1.3 \\
\hline 120 & $2 \cdot 0$ & & & $2 \cdot 1$ \\
\hline 160 & $2 \cdot 8$ & 29 & 29 & $2 \cdot 8$ \\
\hline$\therefore 00$ & $\ldots$ & $3 \cdot 4$ & 34 & \\
\hline 240 & $\ldots$ & 38 & 38 & 3.9 \\
\hline $3: 0$ & $\cdots$ & $\cdots$ & 45 & 4.5 \\
\hline 400 & $\ldots$ & $\ldots$ & 50 & \\
\hline 480 & $\cdots$ & $\cdots$ & 54 & $5 \cdot 3$ \\
\hline $\begin{array}{l}500 \\
640\end{array}$ & $\cdots$ & $\cdots$ & $\begin{array}{l}5.8 \\
6.2\end{array}$ & $5 \cdot 8$ \\
\hline & $\cdots$ & & & \\
\hline
\end{tabular}

TABLE V.

Pressure $\cdot 171$ millim.

\begin{tabular}{|c|c|c|c|c|}
\hline $\mathrm{X}$. & $l=2$. & $l=1$. & $l=\cdot 5$. & $\begin{array}{l}\text { Culeu- } \\
\text { lated. }\end{array}$ \\
\hline 20 & -24 & & & \\
40 & 65 & $\ldots$ & $\ldots$ & $\cdot 66$ \\
80 & $1 \cdot 35$ & $1 \cdot 36$ & $\ldots$ & $1 \cdot 37$ \\
120 & $1 \cdot 8$ & & & \\
160 & $2 \cdot 25$ & $2 \cdot 1$ & $2 \cdot 1$ & $2 \cdot 1$ \\
240 & $\ldots$ & 245 & $\ldots$ & $2 \cdot 4$ \\
320 & $\ldots$ & $2 \cdot 65$ & $2 \cdot 7$ & $2 \cdot 6$ \\
480 & $\ldots$ & $\ldots$ & $3 \cdot 15$ & $2 \cdot 8$ \\
640 & $\cdots$ & $\cdots$ & $3 \cdot 25$ & $3 \cdot 0$ \\
\hline
\end{tabular}

The numbers in the fifth columns of these tables were calculated by the method explained in Section 7 . 
5. We may now proceed to investigate the relation connecting $\alpha, p$, and $\mathrm{X}$, the temperature being constant.

I tet $v$ be the velocity acquired by an ion in travelling freely between two points differing in potential by $\mathrm{P}$ (volts). Let $e$ be the charge on an ion, and $m$ its mass.

Then

$$
\frac{m v^{2}}{2}=\frac{e \times \mathrm{P}}{300} . . . \quad . \quad . .
$$

Let $n$ be the number of molecules in a c.c. of a gas at atmospheric pressure $\left(10^{6}\right.$ in c.G.s. units.), and temperature $20^{\circ} \mathrm{C}$. (which was about the mean temperature at which the experiments were made), and $u$ the mean velocity of agitation of a particle of mass $m$ immersed in a gas at temperature $20^{\circ}$.

The velocity $u$ is given by the equation

$$
\frac{1}{3} m n i^{2}=10^{6} \text {. . . . . . . }
$$

Hence from equations (3) and (4) we obtain

since

$$
\frac{v^{2}}{u^{2}}=\frac{2 n \times e \times \mathrm{P}}{9} 10^{-8}=25 \mathrm{P} \text {. . }
$$

$$
n \times e=1 \cdot 110^{10} \mathrm{q} \cdot \mathrm{p} . *
$$

Hence the velocity acquired by an ion in travelling freely between two points differing in potential by 4 volts, is ten times as great as its velocity of agitation at ordinary temperatures. This result is independent of the mass of the ion.

Under the circumstances with which we are dealing, there is a remarkable difference between the positive and negative ions. The latter produce new ions when moving in a field of force which is too small to maintain a continuous discharge. It is therefore reasonable to suppose that the negative ions with which we are dealing are the same as the negatively charged particles which are given off when ultra-violet light falls on a zinc plate. It has been shown by Professor Thomson that the mass of these particles is $\frac{1}{500}$ of the mass of a molecule of hydrogen $\dagger$. Becquerel and curie bave also shown that the radiation emitted by radium is composed of similar corpuscies.

It seems probable in the present case also that the negative ions are very small, and that the positive ions differ little from ordinary molecules as fur as their mass is concerned. If we adopt this view, it is easy to see that the velocity of

* John S. Townsend, Phil. Trans. rol. cxciii. 1899.

$\dagger$ J. J. Thomson, Phil. Mrg. vol, xlviii. Dec. 1899. 
agitation of the negative ions is 80 times the velocity of agitation of the molecule 3 of air: and the velocity acquired by an ion in travelling between two points differing in potential by 4 volts would be 800 times the velocity of agitation of the molecules of air. The latter velocity is therefore so small in comparison with the velocities of the negative ions, that it may be neglected, and the molecules of the air may be considered to be at rest.

Let us now consider what happens when an ion is constrained to move with a fixed velocity (greater than its velocity of agitation) through a gas at pressure $p$. The number of collisions that the ion makes with molecules of the gas in going through a centimetre is independent of the velocity and is jroportional to the pressure. Let $\beta p$ denote the number of collisions; then $\frac{1}{\beta \mu}$ will be the length of the mean free path, expressed in centimetres. According to our theory, the new ions are produced by collision, and if the velocity with which the ion is constrained to move is sufficiently great, it will produce $\beta p$ new negative ions and an equal number of positive ions, in going through one centimetre of the gas.

Since the collisions are not all of the same kind, an arbitrary velocity of the ion might be sufficient to produce ions on some occasions without producing the maximum number $(\beta p)$. We would expect, therefore, that there is not a fixed minimum velocity of impact necessary to produce ions, but the greater the velocity, the nearer will the number of new ions produced approach the value $\beta p$.

Using these principles as a basis for our theory, we may proceed in the following manner to find an expression for $a$ in terms of $\mathrm{X}$ and $p$.

6. The free paths described by an ion as it moves through a gas will not be all of equal length. Out of $y$ paths the number which exceed the length $x$ is

where $e$ is the mean free path.

$$
y e^{-\frac{x}{c}}
$$

In going along a centimetre an ion has $\beta p$ free paths, so that the number of paths which exceed the length $x$ is

$$
\beta p \epsilon^{-\beta p x} \text {. }
$$

The number of patbs intermediate between $x_{1}$ and $x_{2}$ is

$$
\beta p\left(\epsilon^{-\beta p x_{1}}-\epsilon^{-\beta p x_{2}}\right) \text {. }
$$

Let $I_{P}$ be the velocity acquired by an ion in travelling 
freely between two points differing in potential by $\mathrm{P}$ volts. Let $x_{1} \times \mathrm{X}=\mathrm{P}$ and $x_{2} \times \mathrm{X}=\mathrm{Q}, \mathrm{X}$ being the force acting on the gas.

Then the number of times that an ion collides, with velocities intermediate between $I_{P}$ and $I_{Q}$, in going through one centimetre is

$$
\beta p\left(e^{-\frac{\beta \cdot p \cdot \mathbf{P}}{\overline{\mathbf{X}}}}-\epsilon^{-\frac{\beta \cdot p \cdot \boldsymbol{Q}}{\mathbf{X}}}\right), \text {. . . . }
$$

assuming that after a collision the velocity of the ion is small compared with its velocity before collision. (This assumption would not be legitimate for very large velocities of impact, but the hypothesis may be applied to the velocities with which we are dealing, and leads to simple analysis.)

If the potential $P$ is large, then, according to our theory, a pair of new ions will be formed at each of these collisions, but when $\mathrm{P}$ is small (about 10 or 20 volts) new ions will only be made on some of the more favourable occasions. Let $\dot{\beta_{P}}$ be the number of negative ions formed in $\beta$ collisions when the velocity of impact is between $I_{P}$ and $I_{P+1}$.

Then

$$
\alpha=p \sum_{\mathbf{P}=0}^{\mathbf{P}=\infty} \beta_{\mathrm{P}}\left(\epsilon^{-\frac{\beta p \mathbf{P}}{\bar{X}}}-\epsilon^{-\frac{\beta p(\mathbf{P}+1)}{\mathrm{X}}}\right) \ldots .
$$

The maximum value that any of the coefficients $\beta_{\mathbf{P}}$ can have is $\beta$.

Equation (7) can be expressed in the more general form :

$$
\frac{a}{p}=f\left(\frac{\mathrm{X}}{p}\right) ; \quad \text {. . . . . }
$$

and if no restriction is placed on the form of the function $f$, it is not necessary to assume that the velocity of the ion is small after impact.

We can test whether the values of $a$ which we have determined can be expressed by means of an equation of this form.

If we plot a curve for each pressure, taking as coordinates
$\frac{\alpha}{p}$ and $\frac{\mathrm{X}}{p}$, the five curves should coincide, since they have each the same equation (8).

The points on the accompanying diagrams which are marked 1,2,3,4, and 5, have as coordinates the values of $\frac{a}{p}$ and $\frac{X}{p}$ deduced from Tables I., II., III., IV., and V. respectively. 


\section{Prof Townsend on the Conductivity produced in}

As the variables extend over large ranges, the points are given on two diagrams, figs. 7 and 8 ; one for values of $\frac{\mathrm{X}}{p}$ less than 320 , and the other on a different scale for the larger values of the variables. The positions of the points on the diagrans show clearly that they all lie on the same curve, so that the results of the experiments confirm the result to which we have been led by theoretical considerations.

Fig. 7.

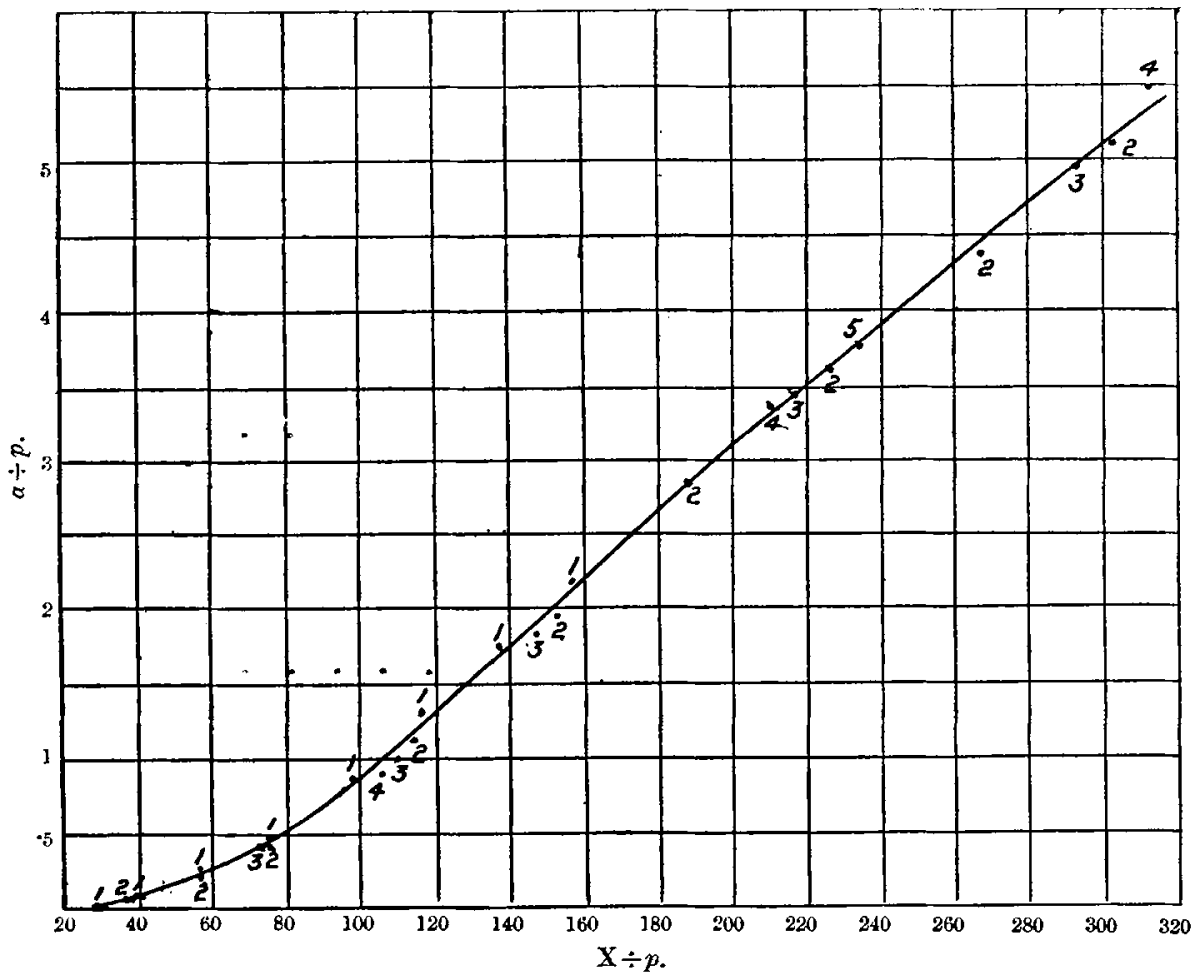

The problem of determining $\alpha$ in terms of $p$ and $\mathrm{X}$ is therefore considerably simplified; and the three variables $\alpha$, $p$, and $\mathrm{X}$ bave been reduced to two : $\frac{\alpha}{p}$ and $\frac{\mathrm{X}}{p}$.

Before we proceed to determine the coefficients in equation 7 we may bere mention an interesting geometrical property of the curve (fig. 8). 
Gases by the Motion of Negatively charged Ions. 213

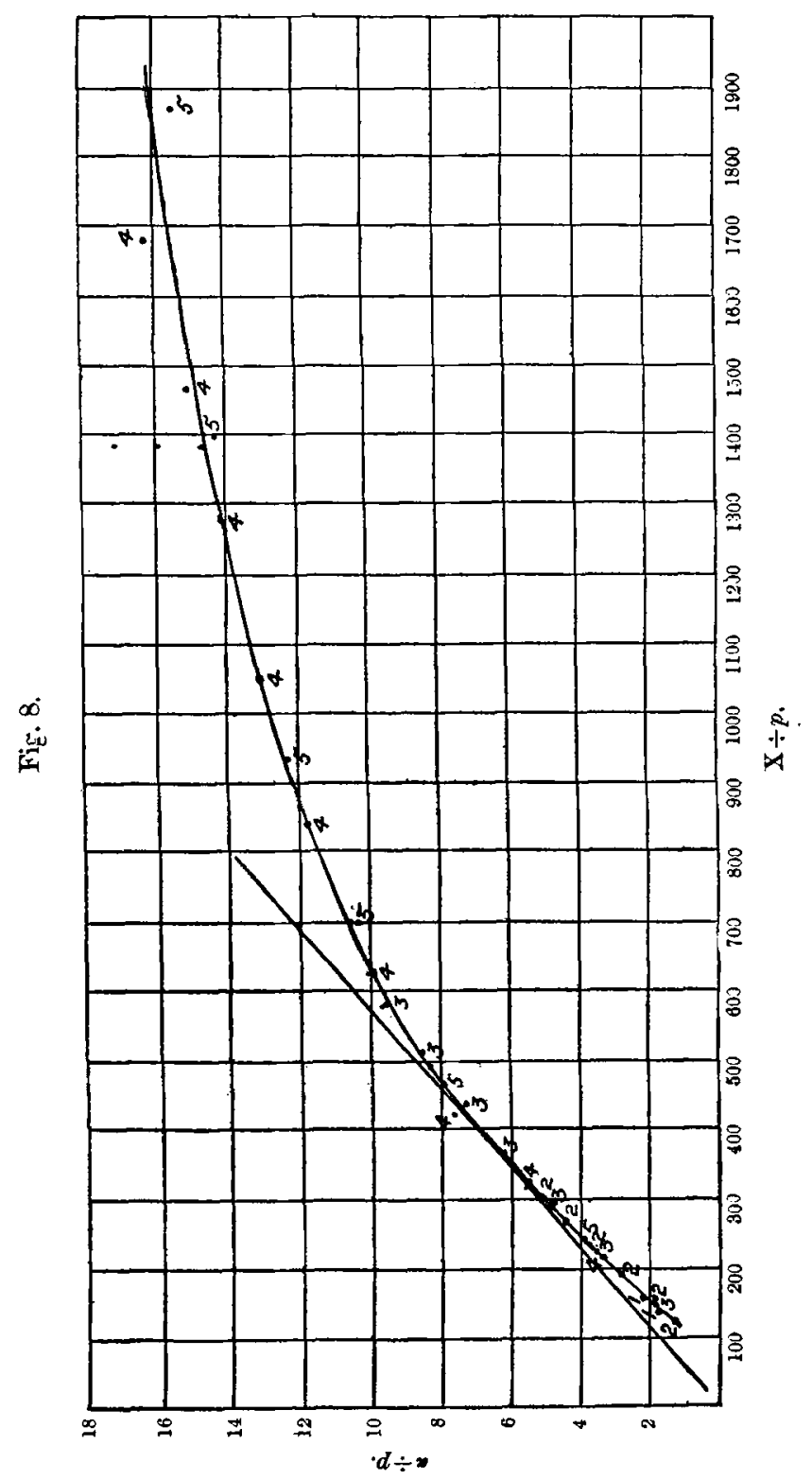




\section{Prof. Townsend on the Conductivity produced in}

When $\mathrm{X}$ is constant, there evidently exists a certain pressure for which $\alpha$ is a maximum : if $p$ is large no new ions will be formed since the original ions never acquire a large velocity, and if $p$ is very small there will be too few colfisions to allow of a large value of $a$.

The value of $p$ for which $a$ is a maximum is obtained by differentiating the equation,

$$
a=p f\left(\frac{\mathrm{X}}{p}\right)
$$

with respect to $p$.

We thus obtain the following equation to determine $p$ in terms of $\mathrm{X}:-$

$$
f\left(\frac{\mathrm{X}}{p}\right)-\frac{\mathrm{X}}{p} f^{\prime}\left(\frac{\overline{\mathrm{X}}}{p}\right)=0 . \quad \text {. . . . }
$$

Since this equation involves $\mathrm{X}$, and $p$, in the form $\frac{\mathrm{X}}{p}$, we conclude that for a given value of $\mathbf{X}$ the value of $p$ which gives the maximum value of $\alpha$ is proportional to $\mathrm{X}$.

The value of $\frac{X}{p}$ which satisfies equation (9) can be obtained immediately from the curve in fig. 8 .

Substituting $\mathrm{X}_{1}$ for $\frac{\mathrm{X}}{p}, \alpha_{1}$ for $f\left(\frac{\mathrm{X}}{p}\right)$, and $\frac{d \alpha_{1}}{d \mathrm{X}_{1}}$ for $f^{\prime}\left(\frac{\mathrm{X}}{p}\right)$ in equation 9 , we obtain

$$
\frac{a_{1}}{\mathrm{X}_{1}}=\frac{d a_{1}}{d \mathrm{X}_{1}}
$$

This relation between the coordinates of a point on a curve $\left[\alpha_{1}=f\left(\mathrm{X}_{1}\right)\right]$ shows that the tangent at the point passes

through the origin.
Hence in order to find the va'ue of $\frac{X}{p}$ which satisfies equation (9) it is only necessary to draw a tangent to the cuve from the origin, and find the abscissa of the point of

contact.
The value of $\frac{\mathrm{X}}{p}$ thus obtained is 380 . Hence the value of $p$ in millimetres for which $a$ is a maximum is $\frac{X}{380}$, where $\mathrm{X}$

The corresponding value of $\alpha$ is

$$
\frac{6.6 \times \mathrm{X}}{380}=.0174 \mathrm{X}
$$

7. Having established the general relation obtained in 
equation 8 , we may simplify the notation by using $\alpha_{1}$ and $\mathrm{X}_{1}$ for $\frac{\alpha}{p}$ and $\frac{\mathrm{X}}{p}$. The new variables denote the values of $\alpha$ and $\mathrm{X}$ when the pressure is one millimetre. When $\mathrm{X}_{1}$ is greater than 400 , the tangent to the curve fig. 7 tends to become parallel to the axis of $x$ when $x_{1}$ is increased. This shows that the value of $\alpha_{1}$ approaches a maximum for large forces.

The greatest value of $\alpha_{1}$ as obtained from the experiments at $\cdot 171$ millim. pressure is 19 . When this value is reached, the increments of $a$ for large increases of force are small, so that the number of collisions $\beta$ cannot much exceed this value. In order to represent the curve by an equation similar to equation 7 , in which the coefficients $\beta_{\mathrm{P}}^{t}$ are less than $\beta$, it was found necessary to take $\beta=21$, which, as we have seen, must be about the correct value.

For the purpose of getting an expression for $\alpha_{1}$ in terms of $X_{1}$ which will agree approximately with the experimental curve, we shall take seven terms in the expansion of $\alpha_{1}$. When the velocity of the ion on impact is less than $I_{5}$, the probability of producing an ion is very small. The number of ions formed by collisions in which the velocity is less than $I_{1}$ may be neglected. Letting $p=1$ we see from equation (6), Section 6 , that the number of impacts per centimetre in which the velocity of the ion is intermediate between $I_{1}$ and $I_{5}$ is

$$
\beta\left(\epsilon^{-\frac{\beta}{X_{1}}}-\epsilon^{-\frac{5 \beta}{X_{2}}}\right) .
$$

Let $b_{1}$ be the average number of new negative ions generated by a single ion in going one centimetre with a velocity intermediate between $I_{1}$ and $I_{5}$. The total number of collisions will be $\beta$ in this cuse. Hence when the ion is moving under an electromotive force $X_{1}$, the number of new ions produced by collisions in which the velocity of impact is intermediate between $I_{1}$ and $I_{5}$ is

$$
b_{1}\left(\epsilon^{-\frac{\beta}{X_{1}}}-\epsilon^{-\frac{5 \beta}{X_{1}}}\right) \text {. }
$$

Hence $\alpha_{1}$ can be expressed by the sum of turms :-

$$
\begin{aligned}
\alpha_{1}=b_{1}\left(\epsilon^{-\frac{\beta}{x_{1}}}-\epsilon^{-\frac{5 \beta}{x_{1}}}\right) & +b_{2}\left(e^{-\frac{5 \beta}{\bar{x}_{1}}}-\epsilon^{-\frac{109}{\bar{x}_{1}}}\right) \\
& +b_{8}\left(e^{-\frac{10 \beta}{x_{1}}}-e^{-\frac{15 \beta}{x_{1}}}\right)+\& c .
\end{aligned}
$$




\section{Prof. Towrsend on the Conductivity produced in}

In order to obtain values of $\alpha$ for the range of forces that has been used, it is necessary to find 7 coefficients in equation (10). When $\beta$ is taken equal to 21 , the coefficients so found will be less than $\beta$, and, according to the theory, it is necessary that the coefficients should satisfy this condition.

When the following values of $b$ are substituted in (10), the equation will represent very accurately the curve through the points in figs. 7 and 8 .

$$
\begin{aligned}
& b_{1}=.03 \text { when the velocity of impact } \\
& b_{2}=.75 \text { is intermediate between } I_{1} \text { and } I_{5} \\
& b_{3}=2 \cdot 7 \\
& b_{4}=91 \cdot 0 \\
& b_{n}=13 \cdot 0 \\
& b_{6}=16 \cdot 0 \\
& b_{7}=20 \cdot 0
\end{aligned}
$$

\begin{tabular}{|c|c|c|c|}
\hline " & " & & , \\
\hline "' & ", & $I_{10}$ & ", \\
\hline " & , & $I_{15}$ & ' \\
\hline$"$ & $"$ & $I_{20}$ & ", \\
\hline & ïies over & & "' \\
\hline
\end{tabular}

The values of $a$ have been calculated for the different pressures from equation (10), by replacing $\frac{\alpha}{p}$ and $\frac{\mathrm{X}}{p}$ for $\alpha_{1}$ and $\mathrm{X}_{1}$, and taking the above values of the coefficients. The results are given in the fifth column of the tables in Section 4.

We have thus obtained results which enable us to find approximately the number of ions that wonld be generated by a single ion in moving through a gas with a fixed velocity. The maximum number is $21 \times p$ per centimetre, which correspends to large velocities. The number is $13 \times p$ when the velocity is about $I_{22}$, and for velocities of the order $I_{5}$ tho number is comparatively small.

8. The mean free path which we have deduced can be shown to agree with what we should expect from physical considerations of a more familiar kind.

The mean free path of a molecule of air at pressure $760 \mathrm{~mm}$. and temperature $0^{\circ} \mathrm{C}$., is $* 0.96 \times 10^{-5}$ centim.

We may therefore take the mean free path in air at $20^{\circ} \mathrm{C}$. to be $1.03 \times 10^{-5}$.

The free path here has not quite the same meaning as the free path that we have been considering-that of an ion travelling through a gas supposed to be at rest. The formula used in finding the mean free path from the coefficients of viscosity takes into account the fact that all the molecules are in motion. The collisions that a single molecule would make with other molecules would be less frequent if the

* Meyer, Kinetic Theory of Gases. 
latter were at rest. The mean free path of a molecule travelling in an atmosphere in which the molecules are at rest would be greater in the ratio $\sqrt{2}=1.41$ than the fres: path when all the molecules are moving with their velocities of agitation *. We therefore conclude that a molecule of air moving with a great velority through a centimetre of air at 1 millimetre pressure would make $90 \cdot 7$ collisions.

An ion under similar circumstances makes 21 collisions. Hence the ratio of the free paths is $4 \cdot 3: 1$. This bears out the conclusion to which we were led from other considerations, that the negative ion is small compared with a molecule. If we disregard the charge on the ion and consider that it is a material particle whose dimensions are small compared with those of a molecule, its free path would be four times the free path of a molecule. The ratio which we have obtained $4 \cdot 3: 1$ shows that the number of collision: made by an ion, estimated according to the theory of viscosity of gases, would be $7 \mathrm{per}$ cent. greater than the number of collisions in which it is possibie for new ions to be formed.

Let $2 \mathrm{~S}$ be the distance between the centres of two molecules of air when a collision ocsurs, and let $d$ be the distance between the centre of a molecule and the centre of an ion when new ions are formed.

Then

$$
\frac{4 S^{2}}{d^{2}}=\frac{4 \cdot 3}{1}
$$

Hence

$$
d=.96 \mathrm{~S} \text {. }
$$

It is usual to assume that $\mathrm{S}$ is the radius of the sphere of action which surrounds a molecule, and that the radius of the molecule is much smaller. If we adopt this view, it will be found difficult to explain the results at which we have arrived. It would appear from the above value of $d$ that new ions are made when the original ion comes inside the sphere of action of a molecule, provided that the velocity of the ion is sufficiently great. The effect of a collision under these circumstances ought to diminish as the velocity of the ion is increased ; the experiments, on the contrary, show that the production of ions increases as the velocity is increased.

It therefore appears from the results of the present experiments that some part of the molecule itself must extend to a distance $S$ from the centre, in order that an ion should produce two new ions when $d=\cdot 96 \mathrm{~S}$.

* Maxwell, Phil. Mag. xix. (186j).

Phil. Mag. S. 6. Vol. 1. No. 2. Feb. 1901. 
We can find $\mathrm{S}$ from the present experiments since it is practically the same as $d$.

The number of collisions made by an ion in going through a centimetre of air at one millimetre pressure is : $\frac{\pi \mathrm{S}^{2} \times n}{760}=21$, $n$ being the number of molecules in a c.c. at atmospheric pressure. Taking for $n$ the value $2 \times 10^{19}$, we obtain $\mathrm{S}=1 \cdot 6 \times 10^{-8 *}$.

I may mention that the mean free path of an ion in air is shorter than its mean free path in hydrogen, but as the experiments with this gas are not yet completed, I must reserve the account of them for a future paper.

The number of ions produced in a gas by a single ion moving rapidly through it could be easily found by another method. It has been shown that the charge on the corpuscles emanating from radium can be measured $t$, when a highly active specimen of the substance is used. The ratio of the ionization produced per centimetre of a gas to the charge carried by the corpuscles would give the number of ions produced by each corpuscle in travelling through a centimetre.

I have made experiments with a view to finding the above ratio, but the specimen of the radio-active substance at my disposal was so weak that it was impossible to detect its charge although a very sensitive method was employed. The experiments, however, showed distinctly that each corpuscle in travelling through a centimetre of air at atmospheric pressure produces at least 10,000 ions. According to the theory which I have described, the number should be $760 \times 21$, il the absorption of the radiation by the gas be neglected. I hope to be able to repeat this investigation with some highly active radium and obtain an accurate result.

9. I have already mentioned that the conductivity between the two parallel plates is altered when the electromotive force is reversed. The difference between the currents in the two directions is a direct consequence of an effect discovered by Curie and Sagnac $\ddagger$. They found that the secondary rays, which are given out when Röntgen rays fall on a metal, carry a small negative charge. It would therefore appear that the ionization produced by secondary rays is due to charged corpuscles emitted by the metal. The bodies which produce the greatest secondary ionization also emit the largest charges. 'lhus the brass

* John S. Townsend, Phil. Trans. vol. cxciii. (1899).

+ M. P. Curie et Mme. Curie, Comptes Rendus, vol. cxxx., 5th March, 1900.

I P. Curie et G. Sagnnc, Comples Rendus, vol. cxxx., 9th April, 1900. 
plate in my experiments gives out more negative corpuscles than the aluminium plate through which the Röntgen rays pass.

The corpuscles emitted by the brass plate have an effect upon the conductivity between the two plates which we shall now proceed to investigate.

Let $\mathrm{C}_{\mathbf{I}}$ be the current when the lower plate is positive, and $\mathrm{C}_{2}$ the current with the same electromotive force when the lower plate is negative.

Let $D$ be the number of negative corpuscles emitted from the brass plate, and $\mathrm{C}$ the number of ions (positive or negative) produced between the plates in the volume of the gas.

Then

$$
\begin{aligned}
& \mathrm{C}_{1}=\mathrm{C}+\mathrm{D}, \\
& \mathrm{C}_{2}=\mathrm{C}-\mathrm{D} .
\end{aligned}
$$

Hence $\mathrm{C}=\frac{\mathrm{C}_{1}+\mathrm{C}_{2}}{2}$, so that the correct value of $\mathrm{C}$ is obtained by taking the arithmetic mean of the two currents. The experiments at 171 millim. pressure give $C_{t}=7 \cdot 6$, and $\mathrm{C}_{2}=4 \cdot 6$ when the plates are one centimetre apart and differ in potential by 10 volts. The corresponding numbers for 334 volts are 30 and 27.5 . In the experiments at higher pressures the ratio of $\mathrm{D}$ to $\mathrm{C}$ is smaller.

The negative corpuscles $D$ are probably emitted with different velocities, so that when the pressure is increased, some of them are stopped by the gas before they get to the aluminium plate. It is evident from the experiments at $\cdot 171$ millim. pressure that an electromotive force of 334 volts has little effect in stopping them, since the currents differ by the same amount for high and low potentials. When some of the corpuscles which start with a small velocity are stopped by the air at higher pressures, the equation $C_{2}=C-D$ does not hold accurately, for although $D$ corpuscles start from the plate, some of them are stopped by the gas and driven back to the plate by the electromotive force. In these cases the mean current does not differ much from either of the currents $\mathrm{C}_{1}$ or $\mathrm{C}_{2}$, so that the equation $\mathrm{C}=\frac{\mathrm{C}_{1}+\mathrm{C}_{2}}{2}$ represents with sufficient accuracy the number of ions produced in the volume of the air.

At $4 \cdot 13$ millim. pressure $\mathrm{C}_{1}=37 \cdot 5$ and $\mathrm{C}_{2}=41 \cdot 5$, when the potential-difference between the plates was 20 volts. The correct value of $\mathrm{C}$ probably does not differ from 39.5 by as much as 2 per cent.

When the force is increased and larger conductivities are obtained, the ratio of $\mathrm{D}$ to $\mathrm{C}$ diminishes.

Q 2 
The distribution of the ionization produced by the rays between the two plates is not quite uniform, which gives rise to small differences in conductivity when the forces are larise. The errors arising from this effect are also eliminated by taking the mean of the two conductivities.

The large difference in conductivity which can be obtained by reversing the electromotive force, when one electrode surrounds the other, is not due to either of the causes mentioned in this section. The explanation of this result is given in Section 12.

10. An estimation of the energy required to ionize a molecule can be dedueed from the results ubtained in Section 7. When an ion collides with a molecule, two new ions are produced if the velocity before the impact is sufficiently great. It has been shown that new ions are formed when the velocity is cqual to $I_{5}$. The kinetic energy of an ion moving with this velacity is $\frac{e \times 5}{300}=10^{-11}$ ergs ; and we conclude that the energy required to produce two new ions is not greater than this amount.

An estimation of the energy required to ionize a molecule has also been made by Prof. Rutherford *, and he concluded that the energy required was equal to the work done in moving an ionic charge through 175 volts. If this number is correct, we should not have got any definite increase in the conductivity between two plates by increasing the force, until the difference of potential between the plates considerably exceeded 175 volts. It would therefore appear from the present experiments that this estimate of the energy is much too large.

11. In the short account of the present theory which was published in ' Nature,' I mentioned that the results obtained by Stoletow + , in connexion with the conductivity produced by ultra-violet light, could be explained on the collision theory. We shall examine these results in detail and show that they all agree with our previous conclusions Stoletow investigated the conductivity between two parallel plates, and found how it varied with the pressure of the air, the distance between the plates, and the electromotive force.

The following results were obtained by Stoletow :-

1. When the jressule and force were constint, the conductivity increased with the distance between the plates.

- E. Rutherferd, Proc. Roy. Suc. vol. Jxvii., 14th Nov. 1900.

$t$ Stoletow, Juurnal de Physique, série 2, volix. 
2. When the pressure and distance between the plates were constant, the current increased with the electric force; at very low pressures the current reached a maximum, and remained constant for large forces *.

3. When the electrie force was constant, the conductivity attained a maximum value for a certain critical pressure, which was accurately proportional to the force and independent of the distance between the plates.

If we suppose that a certain number of negative ions are supplied initially by the action of the light on the metal surface, we can explain all these results by the theory which we have been considering. Stoletow's experiments at low pressures show that the number of ions supplied initially cannot vary much with the pressure. For a given force the conductivity would therefore be a maximum for the same value of $p$ as makes $a$ a muximum.

We see therefore that the third result relative to the critical pressure agrees with the conclusion arrived at in Section 7 relative to the maximum value of $\alpha$, and moreover a remarkable numerical coincidence exists between the value of $\frac{\mathrm{X}}{p}$ for which $\alpha$ is a maximum, and the value of $\frac{\mathrm{X}}{p}$ which gives the maximum current with ultra-violet light.

The following table is given by Stoletow :-

$\mathbf{E}$ is the electromotive force between the two plates, the unit being the eleetromotive force of a Clark cell.

$l$ is the distance between the plates in millimetres.

$p$ is the pressure for which the current is a maximum.

The values of $\frac{p l}{\mathrm{E}} 10^{4}$ for the different experiments are given in the fourth column.

$\begin{array}{rccc}\mathrm{E}(\mathrm{Cl} .) . & l \text { (millim.). } & p \text { (millim.). } & \frac{p l}{\mathbf{E}} 10^{4} . \\ 165 & \cdot 25 & 25 \cdot 3 & 383 \\ \mathbf{1 6 5} & \cdot 47 & 13 \cdot 5 & 384 \\ 65 & \cdot 47 & 5 \cdot 3 & 383 \\ 100 & \cdot 83 & 4 \cdot 7 & 389 \\ 65 & \cdot 83 & 3 \cdot 0 & 383 \\ 60 & \cdot 83 & 2 \cdot 8 & 386 \\ 65 & 1 \cdot 91 & 1 \cdot 3 & 382 \\ 65 & 3 \cdot 71 & \cdot 67 & 382 \\ 40 & 3 \cdot 60 & \cdot 43 & 387\end{array}$

- This corresponds to the results obtuined at $\cdot 171$ millim, pressure, as can be seen from the curves, fig. 6. 
When $\mathrm{E}$ is expressed in rolts and $l$ in centimetres, $\mathrm{I}$ find from the above numbers that the mean value of $\frac{\mathrm{E}}{l_{p}}$ is 372 . So that if $\mathrm{X}$ is the foree in volts per centimetre, the valne of $p$ for which the conductivity is a maximum is $p=\frac{\mathrm{X}}{372}$.

In Section 7 we found that the value of $p$ for which $\alpha$ is a maximum when $\mathrm{X}$ is constant is given by the equation $p=\frac{\mathrm{X}}{380}$, so that the empirical law discovered by Stoletow is completely explained by the theory of collisions.

The other results obtained by Stoletow can also be explained, but the numerical coincidence is not very accurate in some cases. The diserepancies occur when the conductivity between the plates is very small, and it is possible that the experimental results are not so accurate in these cases, but on the whole there is a very good agreement between theory and experiment.

Let us consider how the current depends on the distance between the plates when the conductivity is produced by ultra-violet light. The following tablo gives some of the results obtained by Stoletow when the difference of potential between the plates was proportional to their distance apart $l$. For the largest distance (1.08 millim.) the potential was $165 \times$ 1.43 volts.

\begin{tabular}{|c|c|c|c|c|}
\hline$p$ & $l=1.08$ & 655 & $\cdot 393$ & '362 \\
\hline 750 & $i=7.62$ & $7 \cdot 41$ & $7 \cdot 39$ & $\tau \cdot 33$ \\
\hline 69 & $18: 37$ & $17 \cdot 99$ & $17 \cdot 82$ & 16.76 \\
\hline $7 \cdot 7$ & 491 & $112 \cdot 7$ & $48 * 2$ & $32 \cdot 7$ \\
\hline
\end{tabular}

For the larger pressures an increase in the distance between the plates has little effect on the current; but for the pressure $7 \cdot 7$ millims. the current becomes very large when the distance between the plates is increased. If we suppose as before that the ions created by the action of the light start from the plates, then the current should satisfy the equation (1), Section 4,

$$
\mathrm{N}=\mathrm{N}_{0} \boldsymbol{\epsilon}^{a l},
$$

where $l$ denotes the distance between the plates, and $\alpha$ is a constant. 
Gases by the Motion of Negatively charged Tons. 223

Taking $a=34 \cdot 1$ it will be found that the following numbers are proportional to $\mathrm{N}$ when the distances $l$ given in the above table are expressed in centimetres:-

$$
491,117,48 \cdot 2,31 \cdot 9,
$$

which agree with Stoletow's values of the current when the pressure is $7 \cdot 7$ millims.

The elertric force $\left(X=\frac{\mathrm{E}}{l}\right)$ used in these experiments was 2186 volts per centimetre; and although no forces exceeding 800 volts per centimetre wers used in iny experiments, nevertheless the value of a calculated from the curve given in Section 7 is in good agreement with the above number.

Thus $\mathrm{X}_{1}=\frac{2186}{7.7}=284$, the corresponding value of $\alpha_{1}$ deduced from the curve is $4 \cdot 8$, so that $\alpha=36 \cdot 9$.

In order therefore to explain the variations in current which occur for different distances between the plates when $\mathrm{X}$ is constint, it is sufficient to assume that the number of ions given oft' by the action of the light is independent of the distance between the plates, and that other ions are produced by collisions *.

For pressures lower than 70 millims. it would appear from Stoletow's experiments that the number of ions generated by the action of the light is practically constant, but at higher pressures the number of ions given off by the plates seems to diminish. Thus the conductivity at 750 millims. is about half the conductivity at 69 millims. when the force is 2186 volts per centimetre; and since the current is practically independent of the distance between the plates, this effect cannot be explained by the collision theory.

The following considerations, however, show that at a pressure of 750 millimetres, the force of 2186 volts per centimetre is not sufficient to bring all the ions that are generated at the surface into the gas away from the plate. When the ions are set free by the light at the surface of the plate, they tend to move in all directions with their velocities of agitation; and unless the force acting on them is large enough to give them a velocity away from the plate greater than the velocity of agitation, some of them may, after colliding with molecules, come into contact with the plate and be discharged.

The mean free path of an ion in air at atmospheric pressure

* These experiments cannot be explained by the theory of surfacelayers, since the density of ionization is largest at the points furthest from the plate. 


\section{Prof. Townsend on the Conductivity produced in}

is, according to this theory, $4 \cdot 3 \times 10^{-5}$ centimetre. In going through this distance, the ions only acquire a velocity, corresponding to a fall of potential of $2186 \times 4 \cdot 310^{-5}(=\cdot 1)$ volt, which is about 1.5 times the velocity of agitation (Section 5, equation (5)). When the velocity of an ion before impact is nearly the same as the velocity of agitation, the effect of the collision on the ion may reverse the direction of motion without diminishing the velocity. Under the present circumstances it is to be expected that some of the ions move hack towards the plate after colliding with molecules of the gas, and lose their charge by coming into contact with the surface at which they were generated.

In order to ensure that nearly all the ions generated by the light should get into the gas, it would be necessary to have the electric force above a certain value. It would appear from the experiments that a force of 2186 volts per "entimetre would be sufficient for this purpose when the pressure is 69 millims. The fall of potential in a length equal to the mean free path is about a volt in this case, and the velocity acquired is five times the velocity of agitation. Much smaller forces would be necessary for lower pressures in order that the fall of potential in a length equal to the mean free path should be of the order of one volt; thus we have $\frac{\mathrm{X}}{p \times \beta}=1$ or $\frac{\mathrm{X}}{p}=21$. It will be seen from the curve fig. 6
that the value of $\alpha_{1}$ corresponding to the value of $\frac{\mathrm{X}}{p}=21$ is
very small.

Hence if the force $\mathrm{X}$ is constant and the pressure gradually lowered the conductivity increases slowly until $\frac{\mathrm{X}}{p}$ is about 20 ; at this point practically all the ions generated by the light traverse the air-space between the plates. Further diminution in $p$ produces an increase in conductivity owing to the formation of new ions by collisions, the increase being very great for the larger distances between the plates. When $\mathrm{X}$ is about 372 , the value of $\alpha$ is a maximum and $p$ is the critical pressure; for values of $p$ less than $\frac{\mathrm{X}}{37 \mathrm{~L}}$ the number of ions formed by collisions begins to diminish; and finally, when the pressure is very small, the conductivity approaches the value corresponding to $\frac{\mathrm{X}}{p}=20$.

12. We may here compare some of the conclusions at which we have arrived with the results obtained from the coefficients of diffusion of ions. 
The present experiments have led to the conclusion that there is a great difference between the positive and the negative ions at low pressures, and that the negative ions are much smaller than molecules.

At atmospheric pressure the ions diffuse more slowly than the molecules of the gas in which they are generated, so that each ion is associated with a mass larger than the mass of a molecule of the gas. Also, there is no great differences between the rates of diffusion of the positive and negative ions. Subsequent experiments * at pressures virying from 750 to 200 millims. gave no indication of any change in the size of the ions. The slow rate of diffusion of the ions may be explained if we suppose that a number of molecules are collected round the ions, and that the whole group moves about in the gas with the carrier of the charge: the rates of diffusion of the positive and negative ions would depend on the size of the groups accompanying the ions. The particles of matter on which the negative charges reside might be very much smaller than the particles which carry the positive charges, and at the same time their rates of diffusion might be nearly equal.

When the pressure is reduced, and the ions are acted on by forces which cause them to move rapidly through the gas, the groups of molecules which surround the ions probably disappear, and the negative ions move in the gas as if they were accompanied by a mass which is small compared with the mass of a molecule. It is possible that this effect could be produced at any pressure it the electric force was large enough to make the ion move with a velocity greater than its velocity of agitation. It is difficult, however, to arrive at any definite conclusion on this particular point, as our knowledge of the behaviour of ions under the various conditions is very limited.

13. When the current passes between two electrodes, one inside the other, the conductivity is practically unaltered by reversing the current when the electromotive force is small. If the electromotive force is large, the conductivity depends on the direction of the force in a remarkable manner; the current obtained when the inner electrode is positive may be five or ten times as big as the current obtained when the inner electrode is negative. The reason of this can be easily explained by the theory of collisions if we attribute the production of new ions to the negative ions.

* John S. Townserd, Phil. Trans. vol. cxev. 1900. 


\section{Conductivity produced in Gases by Negatively charged Ions.}

The ionization produced by the rays in the space between the electrodes is practically uniform. In order that new ions should be produced by collisions, it is necessary that negative ions should pass through the gas near the inner electrode where the force is large. When the inner electrode is positive, all the negative ions pass through this region and a large conductivity is obtained. When the inner electrode is negative, only a few of the negative ions pass through the space where the force is large, and consequently the conductivity is not rauch increased. It can be easily seen that by varying the shapes of the electrodes, the conductivity may undergo various changes; and by having the inner electrode very small, a phenomenon resembling unipolar conductivity can be obtained.

These results show that the increase in conductivity must be attributed to the negative ions, and that the positive ions do not generate new ions in the cases which we have been considering. We have thus obtained experimental evidence to show that positive and negative ions have very different physical properties.

14. The conclusions which have been arrived at in this paper help to explain some other phenomena connected with the passage of electricity through gases. The cases I have dealt with are probably the simplest; the experiments can be easily arranged so as to test the theory, and furnish data from which we can arrive at definite conclusions.

Professor Thomson * and Dr. H. A. Wilson $\dagger$ have given explanations of some of the phenomena connected with discharge-tubes based upon the suppos tion that ionization is produced by moving ions. In these cases, the phenomena are very complicated, and it is by no means evident that the ionization is principally due to this effect when the electric intensity is small.

Thus in the positive column where the force is of the order of 30 volts per centimetre, the ionization produced by moving ions must be very small as can be seen from the curve fig. 7, which gives the values of a for small forces. Moreover, there is evidence of other effects which produce ionization. It has been found by $\mathrm{E}$. Wiedemann $\ddagger$ that rays are produced in a discharge-tube which are analogous to Röntgen rays, and produce ionization, so that their effect cannot be neglected in the investigation.

If we consider the results of experiments on the potential

* J. J. Thomson, Phil. Mag. September 1900.

† H. A. Wilson, Phil. Mag. June 1900.

$\ddagger$ E. Wiedemann, Zeitschrift für Electrochemie. 
gradient in the positive column, we are again led to conclude that the ionization does not arise principally from collisions. It has been found, in some cases, that the force per centimetre in the positive column is almost independent of the current strength, so that the velocities of the ions would be the same for different currents, and the rate of recombination would be proportional to the square of the current. The rate of production of ions by collisions would be proportional to the current, and the new ions thus produced would not compensate for the loss due to recombination. These and other considerations show that it is impossible to infer from phenomena connected with discharge-tubes that any ionization is produced by collisions when the force is as small as 30 volts per centimetre.

The results which we have obtained show that for a large range of forces, the positive ions do not contribute to the generation of ions, but it is probable that positive ions would produce ionization in stronger fields.

XVIII. On Electric Inertia and the Inertia of Electric Convection. By ARthur Schuster, F.R.S., Professor of Physics at Owcns College, Manchester*.

1. OUR calculations of self-induction are based on the assumption that the currents which traverse a conductor fill it continuously, the flow being treated as that of an incompressible liquid. The assumption is generally recognized not to hold in the case of electrolytes, where electricity is conveyed by a number of irregularly distributed jons. In the immediate neighbonrhood of such an ion, the magnetic field will be many times greater than that calculated on the supposition of continuous distribution, and hence the rotal magnetic energy is underestimated. What is universally recognized in the case of electrolytes must also be conceded when the current is conreyed by a gas, and the idea is gaining ground that even in solid conductors the current consists of moring positive and negative electrons.

It is the object of this paper, to calculate the additional terms which become necessary for the evaluation of selfinduction, and to discuss the possible cases in which the corrections may affect experimental results.

2. I begin by calculating the total energy of a number of electrically charged, equidistant particles, placed in a straight row and all moving with the same speed $(u)$. If the charge (g) of each particle is taken to be spread uniformly over a

* Communicated by the Phyeical Society : read Dec. 14, 1900. 